\title{
Concerning the Stability of BDF Methods
}

\author{
Vanessa Avansini Botta and Messias Meneguette Jr. \\ Faculty of Sciences and Technology, São Paulo State University, Presidente Prudente, SP, Brazil.
}

\begin{abstract}
We present an analysis of $A_{0}$-stability of BDF methods and proof that zero-stable BDF methods are $A_{0}$-stable using the Schur-Cohn criterion. With this result we have that zero-stable BDF methods are stiffly-stable.

Keywords: BDF methods, characteristic polynomials, zeros of polynomials, Schur-Cohn criterion.

PACS: $02.60 . \mathrm{Jh}$
\end{abstract}

\section{INTRODUCTION}

In this paper we present some results about stability of BDF methods and through the Schur-Cohn criterion we proof that zero-stable BDF methods are $A_{0}$-stable. We use some known concepts of stability that can be found in Lambert [1]. First we present the Brown $(K, L)$ methods and the BDF methods, which is one special class of the $(K, L)$ methods, and some results related to the stability of these class of methods. After, the Schur-Cohn criterion is introduced and we use it to analyze the zeros of perturbed characteristic polynomials associated to the BDF methods for $K=1,2, \ldots, 6$. To conclude, we present a section with the conclusions.

\section{BROWN $(K, L)$ METHODS}

The Brown $(K, L)$ methods are defined by

$$
\sum_{i=0}^{K} \alpha_{i} y_{n+i}=\sum_{j=1}^{L} h^{j} \beta_{j} f_{n+K}^{(j-1)}
$$

where the coefficients $\alpha_{i}$ and $\beta_{j}$ are chosen to maximize the precision of the method and are given by

$$
\begin{aligned}
\alpha_{i} & =(-1)^{K-i}\left(\begin{array}{c}
K \\
i
\end{array}\right)(K-i)^{-L}, i=0, \ldots, K-1, \\
\alpha_{K} & =-\left(\alpha_{0}+\alpha_{1}+\ldots+\alpha_{K-1}\right), \\
\beta_{j} & =\frac{(-1)^{j}}{j !} \sum_{i=0}^{K-1}(-1)^{K-i}\left(\begin{array}{c}
K \\
i
\end{array}\right)(K-i)^{j-L}, j=1, \ldots, L .
\end{aligned}
$$

When $L=1$, the $(K, 1)$ class of Brown methods are none other than the well known Backward Difference Formulae (BDF). Further details may be found in Hairer and Wanner [2]. An interesting result in Meneguette [3] concerning the $A_{0}$-stability of the $(K, L)$ method shows that it is related to the polynomial

$$
\rho_{\gamma}(z)=\left(\alpha_{K}+\gamma\right) z^{K}+\alpha_{K-1} z^{K-1}+\ldots+\alpha_{0},
$$

where $\gamma>0$ and

$$
\rho_{0}(z)=\alpha_{K} z^{K}+\alpha_{K-1} z^{K-1}+\ldots+\alpha_{0}=\rho(z),
$$

is the first characteristic polynomial that supplies the zero-stability. That is, if $\rho_{\gamma}(z)$ has all its zeros inside or on the unit circle, a Brown $(K, L)$ method is $A_{0}$-stable.

In Jeltsch [4], we have the following result:

Theorem 1 Brown $(K, L)$ methods are stiffly-stable if and only if they are $A_{0}$-stable and strongly-stable.

CP1048, Numerical Analysis and Applied Mathematics, International Conference 2008

edited by T. E. Simos, G. Psihoyios, and Ch. Tsitouras

(C) 2008 American Institute of Physics 978-0-7354-0576-9/08/\$23.00 
As zero-stable Brown $(K, L)$ methods are strongly-stable (see Meneguette [3]) and if it is possible to prove that the zeros of $\rho_{\gamma}(z)$ lie inside or on the unit circle, we have that zero-stable Brown $(K, L)$ methods are $A_{0}$-stable. Then, to determine a $(K, L)$ method stiffly-stable, we only needed verify the zero-stability. So, Theorem 1 can be written as follow:

Conjecture 1 Brown $(K, L)$ methods are stiffly-stable if and only if they are zero-stable.

Or, equivalently, "Brown $(K, L)$ methods zero-stable are stiffly-stable".

In this paper we analyze only the zeros of characteristic polynomials related to the BDF methods and proof the validity of Conjecture 1 for this class of methods.

\section{SCHUR-COHN CRITERION}

The Schur-Cohn criterion is one classical result of literature, that determines the number of zeros inside the unit circle using conditions on the coefficients of polynomial. More details can be found in Marden [5].

Theorem 2 (Schur-Cohn criterion) If for the polynomial $P(z)=\sum_{i=0}^{n} a_{i} z^{i}$ all the determinants

$$
\Delta_{k}=\left|\begin{array}{ccccccc}
a_{0} & 0 & \ldots & 0 & a_{n} & \ldots & a_{n-k+1} \\
a_{1} & a_{0} & \ldots & 0 & 0 & \ldots & a_{n-k+2} \\
\vdots & \vdots & \ldots & \vdots & \vdots & \ldots & \vdots \\
a_{k-1} & a_{k-2} & \ldots & a_{0} & 0 & \ldots & a_{n} \\
\bar{a}_{n} & 0 & \ldots & 0 & \bar{a}_{0} & \ldots & \bar{a}_{k-1} \\
\bar{a}_{n-1} & \bar{a}_{n} & \ldots & 0 & 0 & \ldots & \bar{a}_{k-2} \\
\vdots & \vdots & \ldots & \vdots & \vdots & \ldots & \vdots \\
\bar{a}_{n-k+1} & \bar{a}_{n-k+2} & \ldots & \bar{a}_{n} & 0 & \ldots & \bar{a}_{0}
\end{array}\right|, k=1,2, \ldots, n
$$

are different from zero, then $P(z)$ has no zeros on the circle $|z|=1$ and $p$ zeros in $|z|<1$, $p$ being the number of variations of sign in the sequence $1, \Delta_{1}, \ldots, \Delta_{n}$ and $\bar{a}_{i}$ the conjugate of complex number $a_{i}, i=0,1, \ldots, n$.

We use the notation $S\left(1, \Delta_{1}, \ldots, \Delta_{n}\right)$ to refer the number of variations of sign in the sequence $1, \Delta_{1}, \ldots, \Delta_{n}$.

\section{ANALYSIS OF CHARACTERISTIC POLYNOMIALS}

In this section we analyze the behavior of the polynomials $\rho_{\gamma}(z)$ related to the BDF methods, for $K=1,2, . ., 6$, using the Schur-Cohn criterion. We consider $\gamma>0$.

Let the characteristic polynomials be related to the BDF methods:

$$
\begin{aligned}
& K=1 \quad \Rightarrow \quad \rho(z)=z+1 \\
& K=2 \quad \Rightarrow \quad \rho(z)=\frac{3}{2} z^{2}+2 z+\frac{1}{2} \\
& K=3 \quad \Rightarrow \quad \rho(z)=\frac{11}{6} z^{3}+3 z^{2}+\frac{3}{2} z+\frac{1}{3} \\
& K=4 \quad \Rightarrow \quad \rho(z)=\frac{25}{12} z^{4}+4 z^{3}+3 z^{2}+\frac{4}{3} z+\frac{1}{4} \\
& K=5 \quad \Rightarrow \quad \rho(z)=\frac{137}{60} z^{5}+5 z^{4}+5 z^{3}+\frac{10}{3} z^{2}+\frac{5}{4} z+\frac{1}{5} ; \\
& K=6 \quad \Rightarrow \quad \rho(z)=\frac{49}{20} z^{6}+6 z^{5}+\frac{15}{2} z^{4}+\frac{20}{3} z^{3}+\frac{15}{4} z^{2}+\frac{6}{5} z+\frac{1}{6} .
\end{aligned}
$$

We will not analyze the zeros of characteristic polynomial of BDF methods for $K \geq 7$ because these methods are zero-unstable, which is not interesting here. 
For $K=1$, we have

$$
\begin{gathered}
\rho_{\gamma}=(1+\gamma) z+1 \text { and } \\
\Delta_{1}=-\gamma^{2}-2 \gamma<0
\end{gathered}
$$

So, $S\left(1, \Delta_{1}\right)=1$. Then, through the Schur-Cohn criterion, $\rho_{\gamma}(z)$ has one zero inside the unit circle.

When $K=2$,

$$
\begin{gathered}
\rho_{\gamma}=\left(\frac{3}{2}+\gamma\right) z^{2}+2 z+\frac{1}{2}, \\
\Delta_{1}=-\gamma^{2}-3 \gamma-2<0 \text { and } \\
\Delta_{2}=\gamma^{4}+6 \gamma^{3}+9 \gamma^{2}+4 \gamma>0 .
\end{gathered}
$$

Then, $S\left(1, \Delta_{1}, \Delta_{2}\right)=2$ and $\rho_{\gamma}(z)$ has all its zeros inside the unit circle.

In the case $K=3$,

$$
\begin{gathered}
\rho_{\gamma}=\left(\frac{11}{6}+\gamma\right) z^{3}+3 z^{2}+\frac{3}{2} z+\frac{1}{3}, \\
\Delta_{1}=-\gamma^{2}-\frac{11}{3} \gamma-\frac{13}{4}<0, \\
\Delta_{2}=\gamma^{4}+\frac{22}{3} \gamma^{3}+\frac{637}{36} \gamma^{2}+\frac{223}{12} \gamma+\frac{15}{2}>0 \text { and } \\
\Delta_{3}=-\gamma^{6}-11 \gamma^{5}-\frac{439}{12} \gamma^{4}-\frac{3121}{54} \gamma^{3}-\frac{547}{12} \gamma^{2}-15 \gamma<0 .
\end{gathered}
$$

So, $S\left(1, \Delta_{1}, \Delta_{2}, \Delta_{3}\right)=3$ and $\rho_{\gamma}(z)$ has all its zeros inside the unit circle.

For $K=4$, we have

$$
\begin{gathered}
\rho_{\gamma}=\left(\frac{25}{12}+\gamma\right) z^{4}+4 z^{3}+3 z^{2}+\frac{4}{3} z+\frac{1}{4} \\
\Delta_{1}=-\gamma^{2}-\frac{25}{6} \gamma-\frac{77}{18}<0 \\
\Delta_{2}=\gamma^{4}+\frac{25}{3} \gamma^{3}+\frac{869}{36} \gamma^{2}+\frac{1669}{54} \gamma+\frac{545}{36}>0 \\
\Delta_{3}=-\gamma^{6}-\frac{25}{2} \gamma^{5}-\frac{1885}{36} \gamma^{4}-\frac{8387}{72} \gamma^{3}-\frac{97499}{648} \gamma^{2}-\frac{11675}{108} \gamma-\frac{100}{3}<0 \text { and } \\
\Delta_{4}=\gamma^{8}+\frac{50}{3} \gamma^{7}+\frac{1475}{18} \gamma^{6}+\frac{12055}{54} \gamma^{5}+\frac{485425}{1296} \gamma^{4}+\frac{384623}{972} \gamma^{3}+\frac{26435}{108} \gamma^{2}+\frac{200}{3} \gamma>0 .
\end{gathered}
$$

Then, $S\left(1, \Delta_{1}, \Delta_{2}, \Delta_{3}, \Delta_{4}\right)=4$ and $\rho_{\gamma}(z)$ has all its zeros inside the unit circle.

When $K=5$, we have

$$
\begin{gathered}
\rho_{\gamma}=\left(\frac{137}{60}+\gamma\right) z^{5}+5 z^{4}+5 z^{3}+\frac{10}{3} z^{2}+\frac{5}{4} z+\frac{1}{5}, \\
\Delta_{1}=-\gamma^{2}-\frac{137}{30} \gamma-\frac{745}{144}<0 \\
\Delta_{2}=\gamma^{4}+\frac{137}{15} \gamma^{3}+\frac{35567}{1200} \gamma^{2}+\frac{36821}{864} \gamma+\frac{60467}{2592}>0 \\
\Delta_{3}=-\gamma^{6}-\frac{137}{10} \gamma^{5}-\frac{229853}{3600} \gamma^{4}-\frac{8656331}{54000} \gamma^{3}-\frac{682597}{2880} \gamma^{2}-\frac{7630151}{38880} \gamma-\frac{362729}{5184}<0, \\
\Delta_{4}=\quad \gamma^{8}+\frac{274}{15} \gamma^{7}+\frac{338081}{3600} \gamma^{6}+\frac{31675523}{108000} \gamma^{5}+\frac{2001866069}{3240000} \gamma^{4}+\frac{700839973}{777600} \gamma^{3}+\frac{2051274053}{2332800} \gamma^{2} \\
+\frac{7960715}{15552} \gamma+\frac{3610}{27}>0
\end{gathered}
$$


and

$$
\begin{aligned}
\Delta_{5}= & -\gamma^{10}-\frac{137}{6} \gamma^{9}-\frac{28759}{240} \gamma^{8}-\frac{754777}{1800} \gamma^{7}-\frac{109858699}{108000} \gamma^{6}-\frac{1811870993}{1012500} \gamma^{5}-\frac{10776525569}{4665600} \gamma^{4} \\
& -\frac{7206136553}{3499200} \gamma^{3}-\frac{17194715}{15552} \gamma^{2}-\frac{7220}{27} \gamma<0 .
\end{aligned}
$$

So, $S\left(1, \Delta_{1}, \Delta_{2}, \Delta_{3}, \Delta_{4}, \Delta_{5}\right)=5$ and $\rho_{\gamma}(z)$ has all its zeros inside the unit circle.

For $K=6$,

$$
\begin{gathered}
\rho_{\gamma}=\left(\frac{49}{20}+\gamma\right) z^{6}+6 z^{5}+\frac{15}{2} z^{4}+\frac{20}{3} z^{3}+\frac{15}{4} z^{2}+\frac{6}{5} z+\frac{1}{6}, \\
\Delta_{1}=-\gamma^{2}-\frac{49}{10} \gamma-\frac{21509}{3600}<0, \\
\Delta_{2}=\gamma^{4}+\frac{49}{5} \gamma^{3}+\frac{12427}{360} \gamma^{2}+\frac{970133}{18000} \gamma+\frac{16554433}{518400}>0, \\
\Delta_{3}=-\gamma^{6}-\frac{147}{10} \gamma^{5}-\frac{43807}{600} \gamma^{4}-\frac{586333}{3000} \gamma^{3}-\frac{1342202383}{4320000} \gamma^{2}-\frac{481943203}{1728000} \gamma-\frac{1011235687}{9331200}<0, \\
\Delta_{4}=\gamma^{8}+\frac{98}{5} \gamma^{7}+\frac{6557}{72} \gamma^{6}+\frac{14061581}{54000} \gamma^{5}+\frac{7068297193}{12960000} \gamma^{4}+\frac{167890022339}{194400000} \gamma^{3}+\frac{150983713447}{155520000} \gamma^{2} \\
+\frac{93274876933}{139968000} \gamma+\frac{141957067}{691200}>0, \\
\Delta_{5}=-\gamma^{10}-\frac{49}{2} \gamma^{9}-\frac{138397}{1800} \gamma^{8}-\frac{5349353}{27000} \gamma^{7}-\frac{227041787}{480000} \gamma^{6}-\frac{362971653349}{388800000} \gamma^{5}-\frac{18045051832441}{11664000000} \gamma^{4} \\
-\frac{9710592369037}{4665600000} \gamma^{3}-\frac{16696247755537}{8398080000} \gamma^{2}-\frac{2368892113}{2073600} \gamma-\frac{93639}{320}<0
\end{gathered}
$$

and

$$
\begin{aligned}
\Delta_{6}= & \gamma^{12}+\frac{147}{5} \gamma^{11}+\frac{10143}{200} \gamma^{10}+\frac{506401}{3600} \gamma^{9}+\frac{137714549}{288000} \gamma^{8}+\frac{3428467469}{3600000} \gamma^{7}+\frac{2797961343149}{1944000000} \gamma^{6} \\
& +\frac{23921237282507}{9720000000} \gamma^{5}+\frac{12085931037439}{3110400000} \gamma^{4}+\frac{50750948659537}{12597120000} \gamma^{3}+\frac{4856767153}{2073600} \gamma^{2}+\frac{93639}{160} \gamma>0 .
\end{aligned}
$$

Then, $S\left(1, \Delta_{1}, \Delta_{2}, \Delta_{3}, \Delta_{4}, \Delta_{5}, \Delta_{6}\right)=6$ and $\rho_{\gamma}(z)$ has all its zeros inside the unit circle.

We can observe that $\Delta_{k}$ are polynomials on $\gamma$ with degree $2 k$.

\section{CONCLUSION}

We proof that, for $K=1,2, \ldots, 6$, zero-stable BDF methods are $A_{0}$-stable, using that the zeros of $\rho_{\gamma}(z)$ lie inside the unit circle. Then, for the BDF methods, the Conjecture 1 is true, that is, every zero-stable BDF method is stiffly-stable. The same analysis can be used when $L \geq 2$ for the Brown $(K, L)$ methods, but as the degree of characteristic polynomial increase, the calculus of determinant is difficult.

\section{REFERENCES}

1. J. D. Lambert, Computational Methods in Ordinary Differential Equation, Wiley, New York, 1973.

2. E. Hairer, and G. Wanner, Solving ordinary differential equations II: stiff and differential - algebraic problems, Springer-Verlag, New York, 1996.

3. M. Meneguette, Multistep multiderivative methods and related topics, Ph.D. thesis, Linacre College, NAGroup-OUCL, Oxford University (1987).

4. R. Jeltsch, and L. Kratz, Numer. Math. 30, 25-38 (1978).

5. M. Marden, Geometry of Polynomials, Amer. Math. Soc., Providence, 1966. 
Copyright of AIP Conference Proceedings is the property of American Institute of Physics and its content may not be copied or emailed to multiple sites or posted to a listserv without the copyright holder's express written permission. However, users may print, download, or email articles for individual use. 